\title{
Analysis of mismatch impact on image rejection ratio for passive polyphase filters
}

\author{
A.D. Martínez-Pérez ${ }^{1}$ (D) | F. Aznar ${ }^{2}$ | G. Royo $^{1}$ | C. Sánchez-Azqueta ${ }^{1} \mid$ S. Celma ${ }^{1}$
}

${ }^{1}$ Group of Electronic Design (GDE), Aragon Institute of Engineering Research (i3A), Facultad de Ciencias, Universidad de Zaragoza, Zaragoza, Spain

${ }^{2}$ Group of Electronic Design (GDE), Aragon Institute of Engineering Research (i3A), Centro Universitario de la Defensa, Zaragoza, Spain

Correspondence

A. D. Martínez-Pérez, Group of Electronic Design (GDE), Aragon Institute of Engineering Research (i3A), Facultad de Ciencias, Universidad de Zaragoza,

Zaragoza, Spain.

Email: adimar@unizar.es

\section{Present Address}

Pedro Cerbuna 12, Facultad de Ciencias

Funding information

MINECO-FEDER, Grant/Award Number: TEC2014-52840-R TEC2017-85867-R

\begin{abstract}
Summary
Passive polyphase filters (PPFs) are useful symmetric RC networks for processing analog quadrature signals. Passive polyphase filters are also used to implement differential-quadrature or quadrature-differential converters. The quality of these quadrature signals is essential to achieve good performance in modern communication systems. However, mismatch effects can produce notable degradation in the PPF frequency response, and this results in an important reduction in quadrature signal quality, being amplitude balance and phase offset notably affected. Both these errors could be summarized and evaluated together considering image rejection ratio as a figure of merit. This work deepens in the analysis of mismatch impact on PPF, studying image rejection ratio degradation for 2 PPF types, and a systematic method is proposed to obtain the worst case of mismatch in PPFs with any number of stages. It has been validated in a 65-nm CMOS technology.
\end{abstract}

\section{KEYWORDS}

image rejection ratio (IRR), mismatch, passive polyphase filter (PPF), quadrature, quadraturegeneration, single side band (SSB), worst case

\section{1 | INTRODUCTION}

A quadrature signal is a compound of 2 components: in-phase (I) and quadrature (Q). Both equal in amplitude but spared by $90^{\circ}$ in phase. Thus, the quality of quadrature could be evaluated from the error in both magnitudes. According to this, amplitude balance $\left(A_{B A L}\right)$ and phase error $(\Delta \theta)$ are defined as the ratio between I and Q amplitudes and the deviation from the ideal $90^{\circ}$ phase offset, respectively.

Both errors affect the image rejection ratio (IRR), defined as the ratio of desired signal power to image signal power in quadrature transceivers. This makes this magnitude an easily evaluable figure of merit of the quadrature accuracy:

$$
I R R=\frac{\text { Desired Signal Level }}{\text { Image Signal Level }}=\frac{1+2 A_{B A L} \cos \Delta \theta+A_{B A L}^{2}}{1-2 A_{B A L} \cos \Delta \theta+A_{B A L}^{2}} .
$$

A passive polyphase filter (PPF) bases its performance on a symmetrical design, ${ }^{1}$ so any mismatch among its components can cause an imbalance that produces an increase in $A_{B A L}$ and $\Delta \theta$. In a typical application, a PPF is used in conjunction with a mixer stage ${ }^{2}$ and a local oscillator ${ }^{3}$ for upconverting or downconverting analog signals while image 
signal is suppressed. This is a critical operation for single side band heterodyne transmitters or receivers, being specially important in those that shall fulfill the requirements of a WiFi alike standard, for which IRR must be above $40 \mathrm{~dB} .{ }^{4}$

However, only an IRR of $20 \mathrm{~dB}$ is obtained if, due to temperature and process variations, there is a change of $20 \%$ in passive components. ${ }^{5}$ In the same way, mismatch seriously limits the passive filter performance, ${ }^{6}$ as it does in active filters based on balanced structures.

In this paper, the effect of mismatching on PPFs is analyzed and evaluated for a nanometer CMOS technology. Section 2 presents a brief description of 2 types of symmetrical PPFs. In Section 3, an analysis of mismatch for the simplest 1-stage case for both PPF types is made. Section 4 focuses on simulating to check analysis results and propose a general method to obtain the worst combination of mismatch for a typical 3-stage PPF. Also, a comparison between the results obtained and a Monte Carlo analysis in a 65-nm CMOS technology is shown. Finally, main conclusions are drawn in Section 5.

\section{I PASSIVE POLYPHASE FILTERS}

A PPF is a symmetric RC network with 4 capacitors and 4 resistors per stage connected in a way that increases IRR (see Figure 1). This property allows their use as differential-quadrature or quadrature-differential converters. The network topology can be understood as a combination of RC and CR filters whose outputs are added constructively or destructively to favor $90^{\circ}$ phase offset and amplitude matching between I and Q output pairs. ${ }^{7}$ However, perfect quadrature, ie, virtually infinite IRR, is only reachable at a single frequency per stage called pole frequency $\left(w_{0}\right)$. The method to cover a range of frequencies above a minimum IRR requirement is by adding more stages. Nevertheless, in that case, signal losses will be increased at a rate of $3 \mathrm{~dB} /$ stage.

According to its input, when the filter is being used to generate the quadrature signal, 2 kinds of PPFs (shown in Figure 2) are found ${ }^{8}$ : type I, whose output has ideal phase offset $\left(\Delta \theta=90^{\circ}\right)$, and type II, where output amplitude balance is perfect $\left(A_{B A L}=1\right)$. Ideally, phase error (in type I) and amplitude balance (in type II) would remain at their ideal values for all frequencies, and the other magnitude error $\left(A_{B A L}\right.$ for type I and $\Delta \theta$ for type II) would be further reduced the closer the signal frequency $w$ is to $w_{0}$. An IRR expression, common to both PPF types, can be expressed as follows:

$$
\operatorname{IRR}(w)=\prod_{n=1}^{N}\left(\frac{w+w_{0 n}}{w-w_{0 n}}\right)^{2}
$$

where $N$ is the number of stages.

Nevertheless, nonidealities could have a great impact on filter response ${ }^{9}$ and reduce IRR. Among these, mismatch is one of the most important. ${ }^{10}$ Process and temperature nonidealities only cause a frequency shifting effect, which has

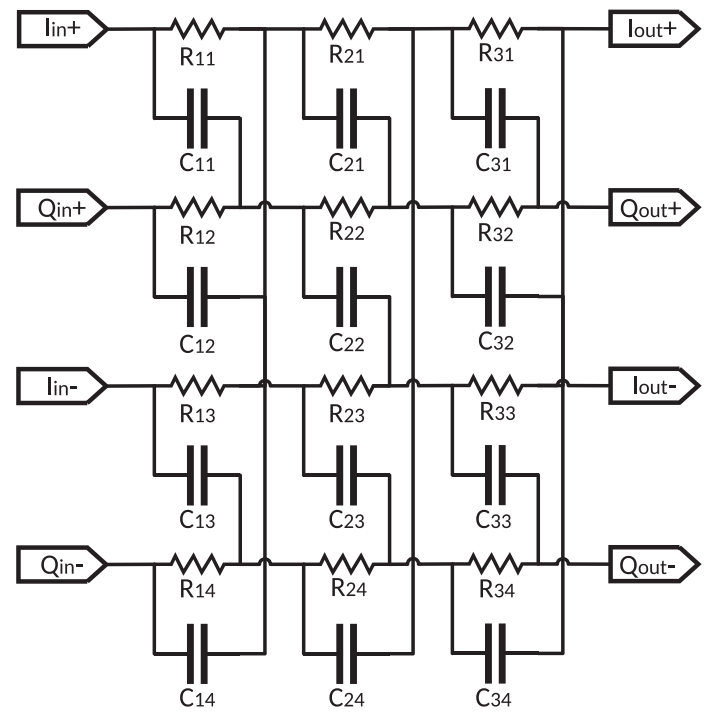

FIGURE 1 Three-stage passive polyphase filter schematic 
FIGURE 2 Types of passive polyphase filters (PPFs): A, Type I generating ideal phase quadrature and B, type II generating unity amplitude balance

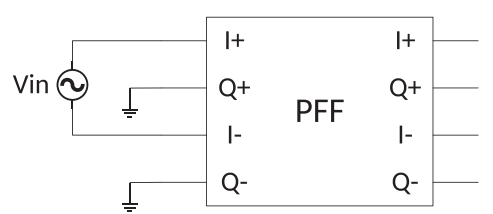

(A)

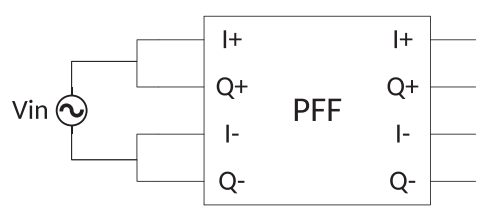

(B)

been previously analyzed in literature. ${ }^{8}$ On the other hand, mismatch could alter the whole filter response, so estimating, or even identifying, the worst-case limit is more difficult. In this work, only mismatch between resistors has been considered, because, in CMOS technology, mismatch between capacitors is generally negligible by comparison. ${ }^{11}$ For instance, in the technology of 65-nm used in this work, MIM minimum-area capacitors present less than $1 \%$ deviation, while $\mathrm{N}+$ polysilicon resistors show a $10 \%$ to $15 \%$ tolerance when they are optimized in area.

\section{3 | MISMATCH ANALYSIS}

An adequate balance between I and Q is the key to a large IRR. Thus, mismatch should have the greatest impact when it causes opposing deviations on I and Q components. This mismatch combination is shown on a 1-stage PPF schematic in Figure 3.

The analysis has been made for a 1-stage PPF in open-circuit output condition; for simplicity and to neglect load effects, negligible because load impedance is much bigger than the one components present. Due to their network topology, multiple stage PPF expressions evaluating mismatch are much larger and less understandable.

Under those conditions and considering voltage in input $I+, Q+, I-$ and $Q-$ as $V_{1}, V_{2}, V_{3}$ and $V_{4}$, respectively, I and $\mathrm{Q}$ values are obtainable as follows:

$$
\begin{gathered}
I=\frac{V_{1}-V_{3}}{j b(1+x)+1}-\frac{j b(1+x)\left(V_{2}-V_{4}\right)}{j b(1+x)+1}, \\
Q=\frac{V_{2}-V_{4}}{j b(1-x)+1}+\frac{j b(1-x)\left(V_{1}-V_{3}\right)}{j b(1-x)+1},
\end{gathered}
$$

where $x$ is tolerance and $b$ is the normalized frequency, defined as $b=w / w_{0}$. According to this topology, $C R=1 / w_{0}$, being $C$ and $R$ nominal capacitor and resistor values, respectively.

\section{1 | Mismatch analysis of type $I$}

In this case, $V_{1}=V_{i} / 2, V_{3}=-V_{i} / 2$ and $V_{2}=V_{4}=0$. This means that (3) and (4) could be expressed as follows:

$$
I=\frac{V_{i}}{j b(1+x)+1} V_{i}=\frac{1-j b(1+x)}{1+b^{2}(1+x)^{2}} V_{i}
$$

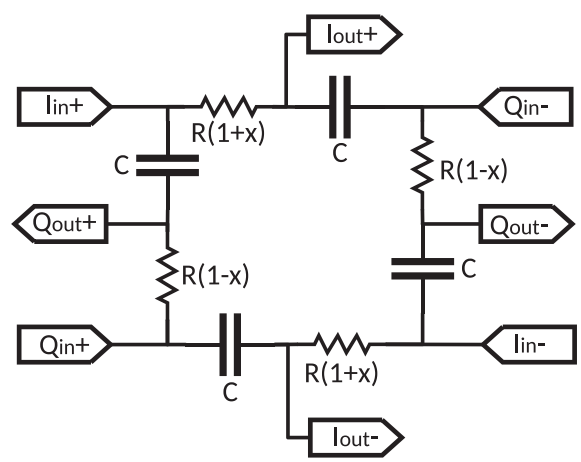

FIGURE 3 Worst-mismatch case 1-stage passive polyphase filter schematic for a given $\pm \mathrm{x}$ tolerance 


$$
Q=\frac{j b(1-x)}{j b(1-x)+1} V_{i}=b(1-x) \frac{b(1-x)+j}{1+b^{2}(1-x)^{2}} V_{i} .
$$

Thus, $A_{B A L}$ and $\Delta \theta$ are as follows:

$$
\begin{gathered}
A_{B A L}=\frac{1}{b(1-x)} \sqrt{\frac{1+b^{2}(1-x)^{2}}{1+b^{2}(1+x)^{2}},} \\
\Delta \theta=-90+\arctan \left(\frac{1+b^{2}(1-x)^{2}}{-2 b x}\right) .
\end{gathered}
$$

Knowing $A_{B A L}$ and $\triangle \theta$, IRR could be obtained from (1). However, it is also possible to define the error caused by $A_{B A L}$ and $\Delta \theta$ separately, which in this case are as follows:

$$
\begin{aligned}
& I R R_{A_{B A L}}=\left.I R R\right|_{\Delta \theta=90^{\circ}}=\left(\frac{1+A_{B A L}}{1-A_{B A L}}\right)^{2}=\left(\frac{1+\frac{1}{b(1-x)} \sqrt{\frac{1+b^{2}(1-x)^{2}}{1+b^{2}(1+x)^{2}}}}{1-\frac{1}{b(1-x)} \sqrt{\frac{1+b^{2}(1-x)^{2}}{1+b^{2}(1+x)^{2}}}}\right)^{2}, \\
& I R R_{\Delta \theta}=\left.I R R\right|_{A_{B A L}=1}=\frac{1+\cos \Delta \theta}{1-\cos \Delta \theta}=\frac{1+\frac{1+b^{2}\left(1-x^{2}\right)}{\sqrt{\left(1+b^{2}(1+x)^{2}\right)\left(1+b^{2}(1-x)^{2}\right)}}}{1-\frac{1+b^{2}\left(1-x^{2}\right)}{\sqrt{\left(1+b^{2}(1+x)^{2}\right)\left(1+b^{2}(1-x)^{2}\right)}}} .
\end{aligned}
$$

In Figure 4, both curves are shown versus normalized frequency and compared with IRR. It can be seen that in the region close to pole frequency, phase error is dominant whereas amplitude balance error dominates in the rest.

When, in (9), the denominator is zero, $I R R_{A_{B A L}}$ would be maximum (virtually infinite). This happens at $b_{\max }$, which is as follows:

$$
1=\frac{1}{b_{\max }(1-x)} \sqrt{\frac{1+b_{\max }^{2}(1-x)^{2}}{1+b_{\max }^{2}(1+x)^{2}}} \rightarrow b_{\max }=\frac{1}{\sqrt{1-x^{2}}} .
$$

The result shows that pole frequency is shifted from 1 to $1 / \sqrt{1-x^{2}}$ because of mismatch. Moreover, a virtually infinite $I R R_{A_{B A L}}$ implies that there is no error due to $A_{B A L}$ at that frequency, so IRR only depends on $\Delta \theta$ and at that frequency the IRR maximum peak is obtained:

$$
I R R_{\text {max }}=I R R_{\Delta \theta}\left(b=b_{\text {max }}\right)=\frac{1+\sqrt{1-x^{2}}}{1-\sqrt{1-x^{2}}}=\frac{\left(1+\sqrt{1-x^{2}}\right)^{2}}{x^{2}} \approx \frac{4}{x^{2}} .
$$

\subsection{Mismatch analysis of type II}

In type-II PPF, $V_{1}=V_{2}=V_{i} / 2$ and $V_{3}=V_{4}=-V_{i} / 2$. Thus, Q and I would be defined as follows:

$$
\begin{gathered}
I=\frac{1-b^{2}(1+x)^{2}-2 j b(1+x)}{1+b^{2}(1+x)^{2}} V_{i} \\
Q=\frac{1+j b(1-x)}{1+j b(1-x)} V_{i}=V_{i},
\end{gathered}
$$




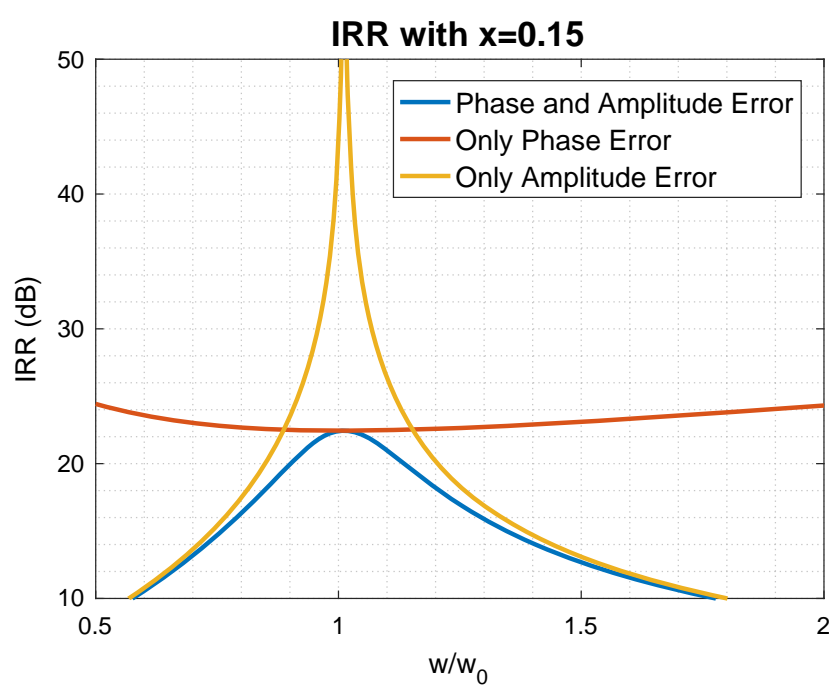

FIGURE 4 Worst-case image rejection ratio (IRR) 1-stage type I passive polyphase filter for a tolerance of 15\%, considering only phase error (orange), only amplitude balance error (yellow), or both (blue) [Colour figure can be viewed at wileyonlinelibrary.com]

and $A_{B A L}$ and $\Delta \theta$ would be

$$
\begin{gathered}
A_{B A L}=1 \\
\Delta \theta=-90+\arctan \left(\frac{-2 b(1+x)}{1-b^{2}(1+x)^{2}}\right) .
\end{gathered}
$$

Equation 15 implies that in type II, mismatch does not alter the ideal $A_{B A L}$ and only $\Delta \theta$ causes error, so IRR is as follows:

$$
I R R \equiv I R R_{\Delta \theta}=\left(\frac{1+b(1+x)}{1-b(1+x)}\right)^{2}
$$

It can be deduced from (17) that the mismatch effect produced in type II is exclusively a frequency shifting: the normalized pole frequency is moved from 1 to $1 /(1+x)$. This displacement is much larger than that caused in type I, although in type II, IRR at the displaced pole is not reduced. A comparison between type I and II is shown in Figure 5.

In addition, comparison reveals that there is a single point $\left(b_{\max }\right)$ where IRR only depends on $|x|$ but not PPF type. Besides, this result is valid even considering capacitive load effects.

\section{4 | WORST-CASE MISMATCH COMBINATIONS}

Obtaining the IRR expression for all possible mismatch combinations or a general equation is hardly practicable even for the 1-stage case. For that reason, most of the time a Monte Carlo analysis is made to check if a design is within desired specifications. However, if it is confirmed that deviations applied in the previous section analysis are, in fact, the worst possible mismatch for a certain tolerance, number of required simulations would be greatly decreased and maximum IRR degradation due to mismatch would be predictable.

It can be easily observed that when mismatch is increased, so is error in $A_{B A L}$ and/or $\Delta \theta$. This has been reported in Galal et al. ${ }^{12}$ Thus, for a given tolerance, $x$, the worst case would be among combinations of 3 possible values: positive maximum deviation $(+x)$, negative maximum deviation $(-x)$, or no deviation (0). This last option should be also considered to avoid error compensation. Unfortunately, the number of possibilities grow exponentially with the number of components involved: for $K$ elements, there will be $3^{K}$ combinations, where $K=4 N$ with $N$ the number of the PPF stages. 


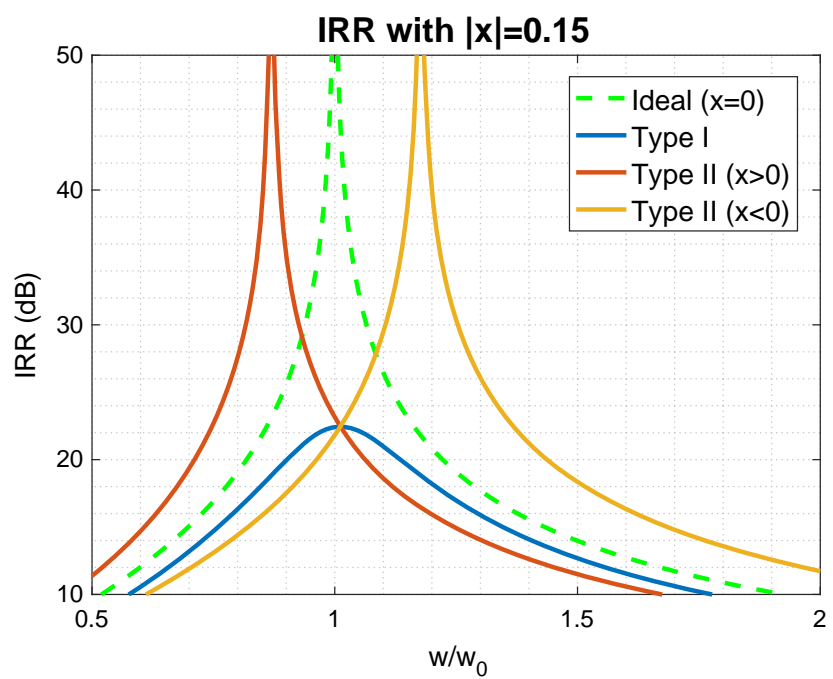

FIGURE 5 Comparison of worst-case filter response variation caused by mismatch in both types for the same deviation (15\%). Type I, blue; type II, orange and yellow [Colour figure can be viewed at wileyonlinelibrary.com]

However, the number of mismatch combinations to test could be reduced. Due to mismatch definition for several elements, deviation in each one could be considered independent. This means that any mismatch combination, including the worst case, could be defined as a $K$-dimensional vector and be spanned by a basis formed by a set of $K$ linearly independent mismatch combinations.

On the other hand, if a basis vector has reduced impact on IRR, it could be deduced that the worst case has no projection on it. In other words, only basis vectors that cause IRR degeneration could span the worst case, so initial sample space could be reduced to that subspace.

According to all these ideas, deviations in resistor values could be described as follows:

$$
\Delta R=x \cdot M_{N} \cdot U
$$

where $\Delta R$ is a column vector containing the deviation of each resistance, $x$ is the tolerance, $M_{N}$ is a $K x K$ matrix for an $N$ stage PPF, which contains the basis, and $U$ is a column vector with those mismatch combination coordinates in $M_{N}$. It should be noticed that in this case $K=4 N$ and all $\Delta R$ elements must be $+x,-x$ or 0 .

In the case of a single stage PPF, 4 vectors would be required to form the basis, $K=4$. The first vector changes all elements in the same amount, while the other 3 linearly independent mismatch combinations are those that fulfill the following conditions: $(a)$ the first element deviation is defined as positive, $(b)$ all deviations are maximum, and $(c)$ the mean of all components together is the nominal value (sum of all deviations is zero). These 4 vectors form $M_{1}$ :

$$
M_{1}=\left[\begin{array}{llll}
m_{11} & m_{12} & m_{13} & m_{14} \\
m_{21} & m_{22} & m_{23} & m_{24} \\
m_{31} & m_{32} & m_{33} & m_{34} \\
m_{41} & m_{42} & m_{43} & m_{44}
\end{array}\right]=\left[\begin{array}{cccc}
+1 & +1 & +1 & +1 \\
+1 & +1 & -1 & -1 \\
+1 & -1 & +1 & -1 \\
+1 & -1 & -1 & +1
\end{array}\right] .
$$

As shown in Figure 6A, it is deduced from simulating results for $M_{1}$ vectors that 2 vectors produce little effect on IRR: $[+1+1-1-1]$ and $[+1-1-1+1]$. Thus, the worst case would be either one of the other 2 basis vectors: $[+1+1+1+1]$ and $[+1-1+1-1]$, or a combination of them. The effect that these basis vectors cause is completely different: while one of them $[+1+1+1+1]$ shifts in frequency, the other $[+1-1+1-1]$ mitigates maximum IRR. As a result, any other combination, while keeping the tolerance, would cause less degradation of IRR. Thus, as supposed, the worst case will be that combination in which I and Q undergo opposite deviations. In Figure 6B, the responses for all $3^{4}=81$ vectors are shown.

For multiple-stage PPF, basis could be built by replicating $M_{1}$ per stage in matrix diagonal and filling the rest with zeros. This proposed method implies that the basis evaluates separately the mismatch contribution of each stage. This generalization has been tested for a typical 3-stage PPF, being $M_{3}$ : 


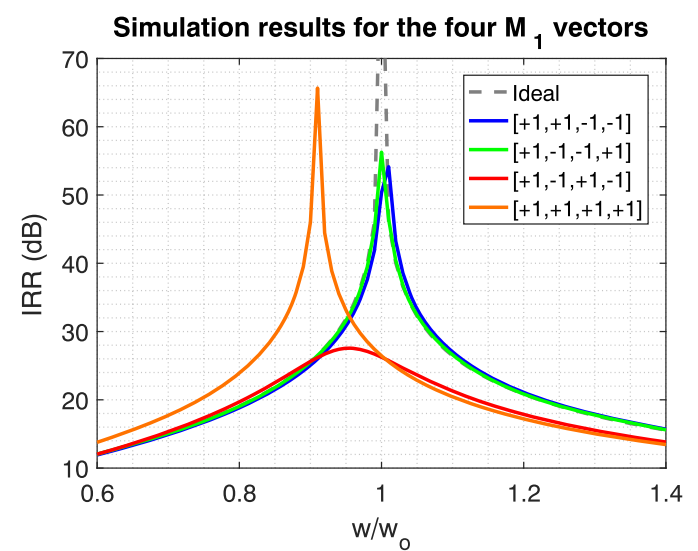

(A)

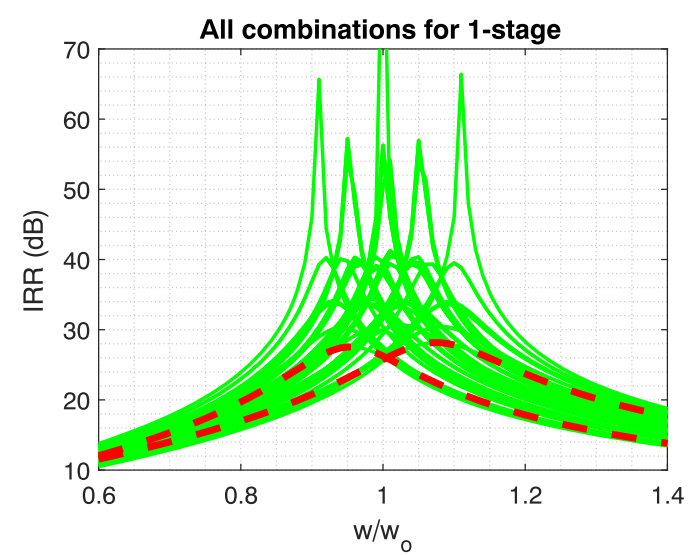

(B)

FIGURE 6 One-stage PPF filter response applying: A, all the different basis $M_{1}$ vectors and B, all 81 possible combinations, where dashed red curves corresponds to the worst cases [Colour figure can be viewed at wileyonlinelibrary.com]

$$
M_{3}=\left[\begin{array}{cccccccccccc}
+1 & +1 & +1 & +1 & 0 & 0 & 0 & 0 & 0 & 0 & 0 & 0 \\
+1 & +1 & -1 & -1 & 0 & 0 & 0 & 0 & 0 & 0 & 0 & 0 \\
+1 & -1 & +1 & -1 & 0 & 0 & 0 & 0 & 0 & 0 & 0 & 0 \\
+1 & -1 & -1 & +1 & 0 & 0 & 0 & 0 & 0 & 0 & 0 & 0 \\
0 & 0 & 0 & 0 & +1 & +1 & +1 & +1 & 0 & 0 & 0 & 0 \\
0 & 0 & 0 & 0 & +1 & +1 & -1 & -1 & 0 & 0 & 0 & 0 \\
0 & 0 & 0 & 0 & +1 & -1 & +1 & -1 & 0 & 0 & 0 & 0 \\
0 & 0 & 0 & 0 & +1 & -1 & -1 & +1 & 0 & 0 & 0 & 0 \\
0 & 0 & 0 & 0 & 0 & 0 & 0 & 0 & +1 & +1 & +1 & +1 \\
0 & 0 & 0 & 0 & 0 & 0 & 0 & 0 & +1 & +1 & -1 & -1 \\
0 & 0 & 0 & 0 & 0 & 0 & 0 & 0 & +1 & -1 & +1 & -1 \\
0 & 0 & 0 & 0 & 0 & 0 & 0 & 0 & +1 & -1 & -1 & +1
\end{array}\right] .
$$

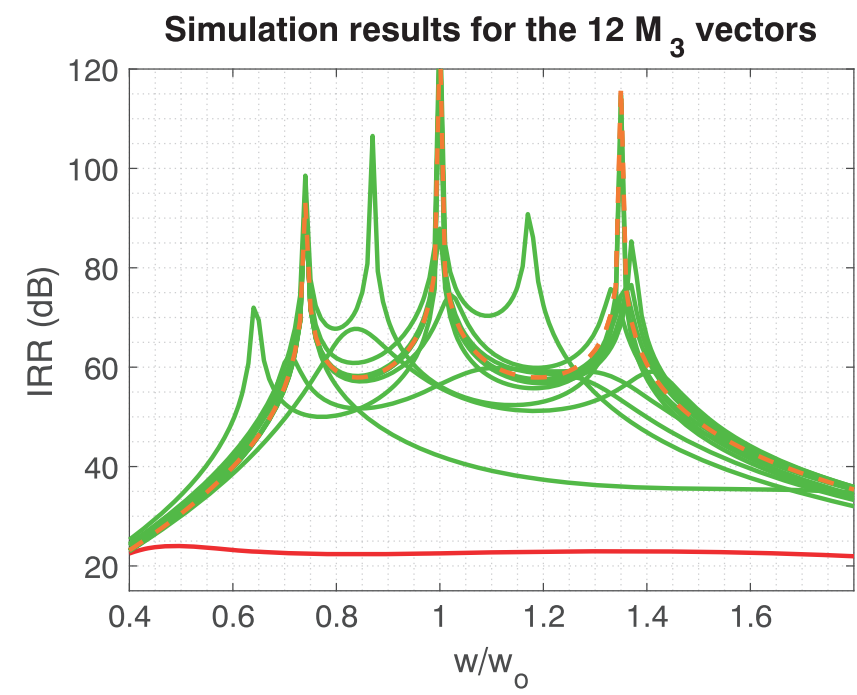

FIGURE 7 Image rejection ratio (IRR) applying all the different basis $M_{3}$ vectors. Orange dashed line describes the ideal response $(x=0)$. The worst case curve is shown in red $[0 \ldots 0+1-1+1-1]$ [Colour figure can be viewed at wileyonlinelibrary.com] 
Simulation results for $M_{3}$ show that IRR degradation is much worse in a basis vector than any others. This is the one that produces opposing deviation between $Q$ and I resistors in the last stage $[0 \ldots 0+1-1+1-1]$ (see Figure 7). Thus, the worst case will be almost equal to that base vector. Figure 8 illustrates this effect: All 256 possible combinations, changing the zero values of the worst vector by maximum deviations $(+x$ or $-x)$, have been simulated for obtaining a representative set. This is shown in red. The same operation has been executed for the 3 following worst cases, although repeated combinations are not considered twice.

To validate our analysis, mismatch effect in a 3-stage PPF has been simulated in 65-nm standard CMOS technology. Loads are capacitors equal to nominal capacitors and mismatch affects the whole filter (resistors and capacitors). Results are shown in Figure 9, where they are compared with the estimated analytical limit according to resistor mismatch. This limit has been easily calculated just by simulating the worst-case vector $[0 \ldots 0+1-1+1-1]$ with positive and negative $x$ in open-circuit output condition.

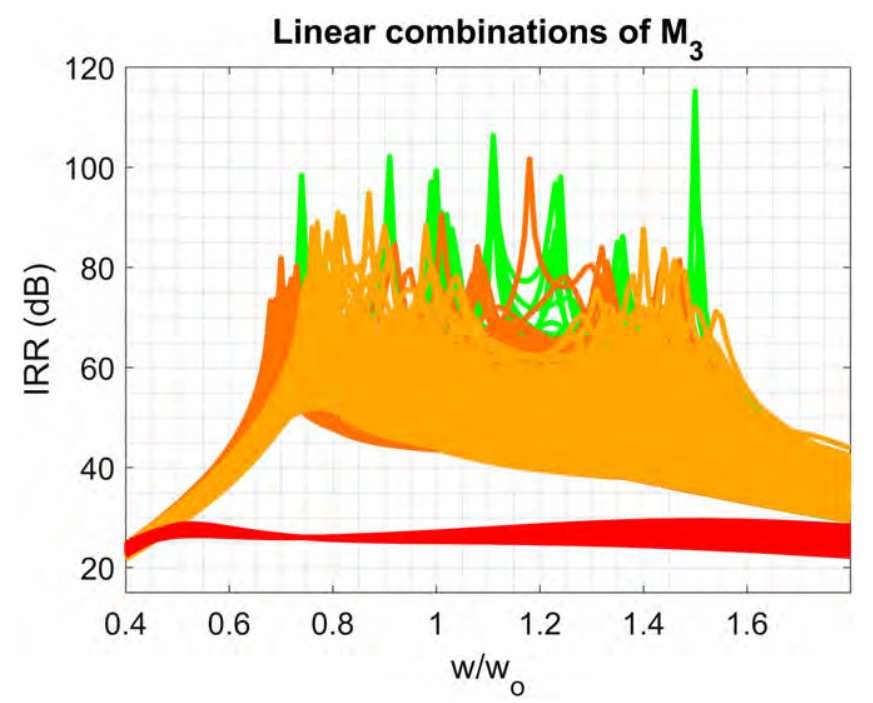

FIGURE 8 In red, the results for the set of 256 possible combinations keeping maximum mismatch between I and Q in the third stage (worst $M_{3}$ vector) and maximum deviation in all elements. In orange, dark orange and green, it is shown the same operation for the following 3 worst $M_{3}$ vectors [Colour figure can be viewed at wileyonlinelibrary.com]

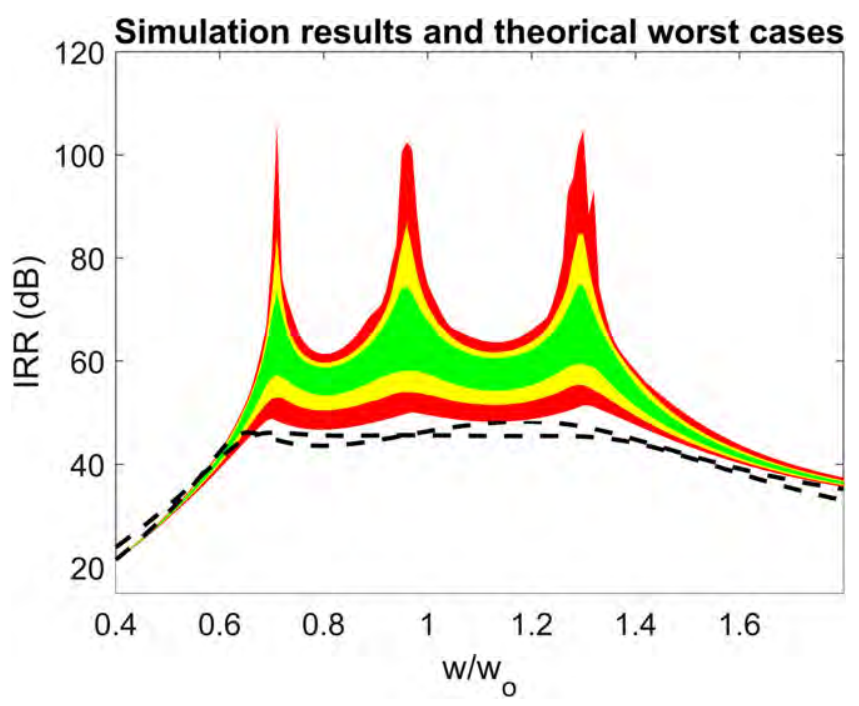

FIGURE 9 In dashed black line, the analytical limit according to resistor mismatch is shown, while the 1000-iteration Monte Carlo simulation results are shown in color. For each frequency, green area comprehends $70 \%$ of the cases, yellow area increases the margins to include $95 \%$, and red zones include the remaining $5 \%$ of cases [Colour figure can be viewed at wileyonlinelibrary.com] 


\section{5 | CONCLUSIONS}

This paper analyzes the effect that mismatch causes on PPF performance. Passive polyphase filters base their operation on a perfect balance among their elements, so mismatch consequently produces a degradation of their frequency response. This estimation supposes a useful tool for a designer because makes it possible to establish a maximum tolerance error in components to guarantee a certain IRR with one single simulation, instead of running hundreds of Monte Carlo simulations.

It has been observed that the worst case is when elements of I and Q branches suffer opposite variations. In the case of multiples stages, if deviation in all of them is similar, mismatch in the last one implies a much greater impact on IRR. In other words, it has been proven that considering the worst mismatch combination possible in only the last stage is a close approximation to the global worst case for this kind of filters.

Furthermore, the effect of mismatching is different depending on the PPF type. While in type I, the main effect is a limitation in maximum IRR value; in type II, a frequency shifting is produced. However, at a given frequency, both types present equal worst-case IRR level, which depends only on tolerance.

It is worth mentioning that although this analysis has been redchecked using a 65-nm CMOS technology, results have a theoretical basis, so they are valid as long as mismatch in capacitors is negligible in comparison with that of resistors.

\section{ACKNOWLEDGEMENT}

This work has been partially supported by MINECO-FEDER (TEC2014-52840-R and TEC2017-85867-R).

\section{ORCID}

A.D. Martínez-Pérez (D) http://orcid.org/0000-0001-5402-1251

\section{REFERENCES}

1. Haddad F, Zaïd L, Rahajandraibe W, Frioui O. Polyphase filter design methodology for wireless communication applications. Mob Wirel Commun. 2010:219-246. ISBN 978-953-307-042-1.

2. Asghari M, Yavari M. Second-order intermodulation cancelation and conversion-gain enhancement techniques for CMOS active mixers. Int J Circuit Theory Appl. 2015;43:1508-1522.

3. Ghonoodi H, Miar-Niami H. Phase error analysis in CMOS injection-coupled LC quadrature oscillator (IC-QO). IntJ Circuit Theory Appl. 2014;42:1123-1138.

4. 802.11-2012, IEEEStd. Part 11: Wireless LAN medium access control (MAC) and physiscal layer (PHY) specifications.

5. Maligeorgos J, Long J. A 2V 5.1-5.8 GHz image-reject receiver with wide dynamic range. In: IEEE Solid State Circuits Conference ISSCC; 2000; San Francisco, CA, USA:322-323.

6. Stuarts G, Julián P. Analysis of time delay difference due to parametric mismatch in matched filter channels. Int J Circuit Theory Appl. 2015;43:1412-1430.

7. Behbahani F, Kishigami Y, Leete J, Abidi AA. CMOS mixers and polyphase filters for large image rejection. IEEE J Solid-State Circuits. June 2001;36(6):873-887.

8. Kaukovouri J, Stadius K, Ryynänen J, Halonen KAI. Analysis and design of passive polyphase filters. IEEE Trans Circuits Syst Regul Pap. 2008;55(10):3023-3037.

9. Martínez-Pérez AD, Morte J, Aznar F, Sánchez-Azqueta C, Celma S. Impact of non-idealities on passive polyphase filter performance. In: 2017 European Conference on Circuit Theory and Design (ECCTD); 2017; Catania:1-4.

10. Shi BE. The effect of mismatch in current- versus voltage-mode resistive grids. Int J Circuit Theory Appl. 2008;37(1):53-65.

11. Hastings A. The Art of Analog Layout. Upper Saddle River, N.J: Prentice Hall; 2001. Ch. 7.

12. Galal SH, Ragaie HF, Tawfik MS. RC sequence asymmetric polyphase networks for RF integrated transceivers. IEEE Trans Circuits Syst II, Analog Digit Signal Process. 2000;47(1):18-27. 
How to cite this article: Martínez-Pérez AD, Aznar F, Royo G, Sánchez-Azqueta C, Celma S. Analysis of mismatch impact on image rejection ratio for passive polyphase filter. Int J Circ Theor Appl. 2018;1-10. https:// doi.org/10.1002/cta.2505 